\title{
Stepwise manipulation of cardiac computed tomography multi-planar reconstruction to mimic trans-esophageal echocardiography
}

\author{
Wei Tsung Lai ${ }^{1}$, Ming-Chon Hsiung ${ }^{1}$, LUNG-CHUN LIN ${ }^{2}$, Wei-Hsian Yin ${ }^{1}$, Ching-Hsuan \\ $\mathrm{Hsu}^{1}$, and Kuan-Chih Huang ${ }^{1}$ \\ ${ }^{1}$ Cheng Hsin General Hospital \\ ${ }^{2}$ National Taiwan University Hospital
}

April 28, 2020

\begin{abstract}
Cardiac computed tomography $(\mathrm{CT})$ is increasingly used to plan transcatheter structural heart interventions, however, intraoperative guidance relies on trans-esophageal echocardiography (TEE) and fluoroscopy. This study sought to develop the methods of stepwise CT multi-planar reconstruction manipulation to mimic TEE thus bridging the gap between preoperative planning and intra-operative guidance tools. This CT manipulation successfully reproduces similar configurations as TEE views in mid-esophageal left ventricle (LV) views, transgastric LV 2-chamber views for mitral apparatus, and other miscellaneous views. Stepwise cardiac CT manipulation to mimic TEE is the final piece of the puzzle in the mental co-registration of these three crucial imaging modalities. With it, we are enabled to foresee the TEE images and fluoroscopy projections in a preoperative rehearsal thus improving the intra-operative accuracy of interventions.
\end{abstract}

\section{KEYWORDS}

Mental co-registration of imaging modalities, multi-planar reconstruction, transcatheter structural heart interventions, interventional echocardiography

\section{1 - INTRODUCTION}

Cardiac computed tomography (CT), fluoroscopy and trans-esophageal echocardiography (TEE) are three major imaging modalities, which support the increasing complexity of trans-catheter interventions in congenital and structural heart diseases. ${ }^{1-3}$ These modalities are based on different mathematical coordinate systems, and are often demonstrated at different visual perspectives. Multi-planar reconstruction (MPR) of cardiac CT, commonly used as pre-operative planning tool, is based on the standard Cartesian coordinate system (Figure 1A). Although manual adjustment is allowed for plane rotation and movement of the origin of the coordinate system into the region of interest (ROI), the re-sliced 2D images can still be intuitively understood because CT datasets include abundant isotropic details in cardiac anatomy. Instead of multi-slice imaging, fluoroscopy provides with the X-ray projection from the visual perspective of the image intensifier, whose trajectory is clinically described by the spherical coordinate system (Figure 1B) as right anterior oblique (RAO)/left anterior oblique (LAO) and cranial (CRA)/caudal (CAU). The coordinate system of TEE is the most complex so the same structure scanned at different esophageal levels requires mental co-registration in understanding (Figure $1 \mathrm{C}$, right). The TEE is basically a cylindrical coordinate system (Figure 1C, left) and its monitor produces 2D anatomical information on the polar coordinate reference plane, similar to the axial and the coronal CT MPR planes for trans-esophageal and trans-gastric windows, 
respectively. However, the longitudinal axis (similar to z-axis in Cartesian system) of such cylindrical coordinate system is not a straight line, not to mention the anteflex and retroflex probe manipulation; rather, the longitudinal axis actually means the esophageal tract. Furthermore, the reference plane is allowed to rotate around its polar axis (the mid-line of the scanning sector) and the geometry of flex to the right/left maneuver is even more complex. As a result, both the acquisition and the understanding of TEE images need learning curve.

Mental co-registration of these modalities is the foundation to facilitate heart-team communication. ${ }^{4,5}$ For quick switch between imaging modalities with different coordinate systems, assistive theory and technology have been developed. Echocardiography-fluoroscopic fusion imaging transforms the coordinate system of TEE probe to that of the image intensifier so that 2D and 3D TEE images can be calibrated and projected onto the $2 \mathrm{D}$ fluoroscopy. ${ }^{6,7}$ As a result, images of these two modalities can be viewed in the same visual perspective ${ }^{4,5,8}$ with acceptable mean target registration error. Piazza and colleagues ${ }^{9,10}$ introduced the concept of optimal projection curve that comprises all the projection angles of which the X-ray tube-to-image intensifier direction is orthogonal to the normal vector of the interested anatomical targets. Their method is applied to minimize the parallax during the deployment of a quasicylindrical device into an anatomical structure of variable geometry. However, the bridge between cardiac CT and TEE is lacking. Adjusting the MPR crosshairs into the center of mitral valve plane and crossing the third MPR line through the LV apex are the traditional methods to obtain LV four-chamber, two-chamber and long-axis CT re-slices without foreshortening. ${ }^{11}$ These views precisely describe the LV segments and mitral apparatus, but are usually at a different reference plane from that of TEE imaging. Although TEE simulator based on CT images has been developed as a valuable teaching tool, ${ }^{12}$ it sacrifices the wide visual field of CT scan and loses image quality during datasets transformation. Due to rapid development of new trans-catheter devices, more and more distinct imaging requirements are called for. As a result, it is mandatory to bridge the gap between the pre-operative planning tool and the intra-operative imaging guidance.

For this purpose, we developed the methods of stepwise cardiac CT MPR manipulation to mimic transesophageal echocardiography. On the other hand, from the patient-specific information of cardiac CT, we can also plan the TEE imaging in a preoperative rehearsal to make the desired standardized TEE views more reproducible.

\section{2 - METHOD (Figure 2)}

Since the motion of TEE probe is confined to the esophagus-stomach tract, it is difficult for TEE sonographers to reproduce the cardiac CT MPR views used for preoperative plan. By contrast, it is easier to use CT datasets to simulate TEE imaging. There are three basic MPR planes - axial, coronal and sagittal - that are perpendicular to each other. One can adjust the cross-sectional orientation of the other two planes by moving and rotating their MPR lines on the selected MPR plane (i.e., we can adjust coronal and sagittal MPR lines on axial plane and vice versa). To represent the TEE scanning sector, we imagine a circular sector with the origin in the MPR crosshairs on the axial plane and the sector has a line of symmetry as the sagittal MPR line. Once we move the MPR crosshairs into the esophagus with the following sequential steps, we can translate basic TEE probe movements into corresponding CT MPR manipulation with the following steps.

Step 1: Manipulation on the sagittal plane: advance, withdrawal, anteflex and retroflex

The first step to simulate TEE on CT axial MPR plane is to invert the z-axis (the coronal MPR line on the sagittal MPR plane), because CT offers a perspective from a caudal direction on the axial MPR plane but TEE, the cranial perspective on the reference plane. Next, we simulate the advance/withdrawal by moving the crosshairs along the esophageal tract into the gastric space. Moreover, we can simulate the anteflex by clockwise rotation of the axial MPR line on the sagittal MPR plane and the retroflex by counterclockwise rotation.

Step 2: Manipulation on the axial plane: turn to the left/right 
Because the sector width of TEE is limited, sonographers have to turn the TEE probe rightward to observe the right-sided structures and vice versa. We can simulate this maneuver by rotating the sagittal MPR line on the axial MPR plane. Step1 and Step 2 can be repeated randomly until satisfactory image is obtained on the axial MPR plane before moving on to the step 3.

Step 3: Manipulation on the coronal plane: rotation, flex to the left/right

Since the z-axis has been inverted at the very beginning, the anterior posterior direction on the coronal MPR plane is reversed, too. As a result, the counterclockwise rotation of TEE scanning plane will be simulated by a clockwise rotation of the axial MPR line on the coronal MPR plane. TEE probe is steerable for delicate adjustment of imaging orientation. The flexible joint is around $5 \mathrm{~cm}$ above the scanning center. To simulate the "flex to the right," two steps have to be taken. Firstly, we rotate the crosshairs about the flexible joint of TEE probe. Secondly, a negative rotation with the same degree that the crosshairs are rotated should be added. Examples of flex to the right at mid-esophagus level and flex to the left at transgastric level are illustrated in Figure 3 and Figure 4 respectively.

\section{3 - RESULTS}

\section{Mid-esophageal LV views without foreshortening (Figure $2 \& 3$ )}

After inverting the z-axis and moving the MPR crosshairs into the esophageal lumen, we simulate "turn to the left" by rotating the sagittal MPR line counterclockwise on the axial MPR plane to make the sagittal plane cross the LV apex. Then we can easily use the sagittal plane to discern that at different level of esophagus, different degrees of retroflex are needed to avoid LV foreshortening. With a LV image without foreshortening, we can rotate the reference plane on the coronal MPR plane to obtain LV 4-chamber, 2chamber, bi-commissural and long-axis images.

Orthogonal interrogation of ultrasound beams obtains the highest reflection to deserve the most precise resolution. As a result, by acquiring the mid-esophageal LV views with the ideal orientation, the imagers can reconstruct the high-resolution 3D mitral valve en face views. However, if the LV axis crossing from the center of mitral valve to LV apex is not contained in the plane of ultrasound scanning beams, there will be configural differences between TEE and CT imaging. On CT TEE simulation, we can easily discern such configural differences by investigating whether the sagittal MPR line on the axial plane crosses the center of mitral valve or not (Figure 3C). Sometimes a little "flex to the right" can partially ameliorate the configural differences. This effect can also be easily realized by CT simulation as a parallel movement of the sagittal line of sector from lateral annulus toward the center of mitral valve on the axial plane. It is important for the operators to understand such configural differences or they will be confused by intraoperative TEE imaging, because the more the configural differences appear, the higher the possibility that the devices make out-of-plane motion on TEE imaging.

\section{Transgastric LV 2-chamber views for mitral apparatus (Figure 5)}

Mitral apparatus evaluation is the key to both surgical and transcatheter mitral valve repair. Severity of leaflet tethering by infarcted myocardium determines the success of mitral repair. During chordal length determination for artificial chordae implantation, it is important to have the papillary muscle, measured chordae tendineae and the edge of mitral valve all be clearly identified simultaneously on a two-dimensional plane in a systolic phase. Such evaluations could be performed on the transgastric LV bi-commissural view, which can be derived from planar rotation from the transgastric LV short axis view at papillary muscle level (Figure 5). On CT simulation, we can start with a mid-esophageal foreshortened 4-chamber view, and then move the MPR crosshairs into the gastric cardia. Next, we simulate anteflex at the sagittal MPR plane to obtain a short axis view at papillary muscle level. Finally, we rotate the axial MPR line on the coronal MPR plane to obtain the transgastric view for mitral apparatus.

Transgastric views for LV outflow tract (LVOT) to ascending aorta (AsAo) (Deep transgastric 5-chamber view and transgastric long axis view) (Figure 6) 
This is the ideal view to obtain a parallel alignment of Doppler signal to the LV outflow jet. When there are aortic or mitral prostheses or calcification, this is the only view to investigate aortic valve cusps without acoustic shadowing. After transcatheter aortic valve replacement, this view is the optimal to evaluate paravalvular leakage; and it is also crucial to estimate the pressure gradient at LVOT and aortic valve after procedures, such as, mitral valve-in-valve and transcatheter aortic/mitral valve replacement. More often than not, there are configural differences between pre-operative CT and intra-operative TEE images on the aorto-mitral structure. On CT simulation, we firstly move the MPR crosshairs into the gastric fundus and then rotate the axial MPR line clockwise toward the aortic valve (step1). Next, we adjust the sagittal MPR line to cross the aortic valve on the axial MPR plane (step2). Lastly, we rotate the axial MPR line on the coronal MPR plane to check the relationship between mitral structure/device and LVOT (step3).

During the TEE manipulation, an important tip to acquire the transgastric LVOT-AsAo view is to add some "flex to the left." The in vitro motion of TEE probe during "flex to the left" deviates somewhat for there is to the certain extent "anteflex" and it becomes, instead, more like a "turn to the left" (i.e., counterclockwise rotation of the TEE probe, Figure 4). However, due to the resistance of the esophageal tract and accumulated torque at esophagogastric junction (EGJ), there will be an in vivo disproportional loss of rotation at the TEE probe to that added by imager. As a result, the "flex to the left" will be a more suitable method to control the TEE probe in the gastric fundus to achieve better contact and a more predictable and controllable rotation. In patients with dilated LV chamber or horizontal AsAo, it is more difficult to acquire the deep transgastric 5-chamber view because the scanning sector cannot simultaneously include the LVOT and LV chamber even with the "flex to the left." In that case, the transgastric long axis view may catch the LVOT-AsAo structure in the right side. If the axial MPR plane cannot produce the ideal deep transgastric 5-chamber view after the step 2 mentioned earlier, (i.e., only AsAo and atria but limited LV chamber), the transgastric long axis view may become a more suitable intraoperative choice (Figure 4). As a result, CT simulation can provide with a quick check for the difficulty in TEE acquisition.

\section{Distal esophageal views for tricuspid valve (Figure 7)}

At the mid-esophageal level, septal leaflet is prone to be attenuated by the acoustic shadowing from interatrial septum; however, such attenuation can be avoided at the distal esophageal level, and 3D en face views reconstructed at this level can be used for delicate investigation in the etiology of tricuspid regurgitation. Although aortic valve disappears sometimes, the anatomical landmarks such as ostium of coronary sinus, Eustachian valve, and rough part of right atrium, can still help determine the orientation in TEE views at the distal esophageal level. On CT simulation, we firstly move the MPR crosshairs into the distal esophageal level, near the EGJ and then rotate the axial MPR line clockwise toward the aortic valve. Next, we simulate "turn to the right" by rotating the sagittal MPR line clockwise on the axial MPR plane to cross the tricuspid valve. Finally, we rotate the axial MPR line on the coronal MPR plane to evaluate the relationship between leaflets.

\section{Transgastric RV inflow views for tricuspid apparatus (Figure 8)}

Transgastric views for right heart give details about moderator band, papillary muscles for anterior and posterior leaflets (RV inlet view), and the only 2D view for simultaneous visualization of all 3 tricuspid leaflets (RV basal view). On CT simulation, we firstly move the MPR crosshairs into the gastric cardia and then rotate the axial MPR line clockwise toward the aortic valve. Next, we simulate "turn to the right" by rotating the sagittal MPR line clockwise on the axial MPR plane to cross the RV. Finally, we rotate the axial MPR line on the coronal MPR plane to produce the RV in-out view and the short axis view of tricuspid leaflets.

Examples of left atrial appendage, short-axis view of aortic valve, and bicaval view of interatrial septum are illustrated in Figure 9.

\section{4 - DISCUSSION}

TEE is the primary image guidance modality used for trans-catheter structural heart intervention tech- 
nologies because of its better real-time visualization of cardiac anatomical structures. However, its limited sector width and complex probe orientation prolong the learning curve to comprehend the spatial configuration of procedural TEE imaging. Although CT has been widely used as a preoperative planning tool for trans-catheter structural heart interventions, the created 2D CT images are usually taken from different visual perspectives of intraoperative TEE images. This creates issues in positioning, docking and taking other specific key steps of these image-guided interventions, because the procedural TEE imaging may fail to reproduce the same prerequisite patterns as the $\mathrm{CT}$ based preoperative planning. Compared to the high degree of freedom in the CT MPR slices, the motion of TEE probe is rather limited. Therefore, the methodology which we propose here is capable of carrying out a preoperative plan by CT MPR to simulate the intraoperative TEE rather than applying the TEE to acquire images conforming to what is showed on CT slices. In this article, we demonstrate a stepwise CT MPR manipulating method to simulate the TEE probe motion and preview the procedural TEE images on a different imaging modality. This reduces somewhat the differences in deciding the size of devices from these two imaging modalities. Besides validating this method in different TEE views, we also applied it on dynamic CT to mimic TEE videos. This concept has not previously been introduced in a systematic fashion.

On the other hand, such a learning curve is also steep for interventional echocardiographers to precisely offer the procedure-specific imaging needs in every patient. ${ }^{13}$ Although basic acquisition protocols of TEE have been introduced by Hahn and colleagues, ${ }^{13-16}$ many patient-specific anatomical conditions, such as clockwise/counterclockwise rotation of the heart, trajectory of esophageal tract, geometry of gastric space and its relative position with cardiac structures all lead to significant differences in the spatial configuration of procedural TEE imaging. As a result, the TEE imaging is of large variability and highly technique dependent. Sometimes, it is even impossible to obtain the ideal procedure-specific views in patients of challenging anatomy. In the application of a traditional method, interventional echocardiographers discern not the feasibility of those procedure-specific views until TEE probe is inserted. Fortunately, in our simulation, the $\mathrm{CT}$ datasets in nature contain all the anatomical information, and thus broaden the vision of interventional echocardiographers beyond the scanning sector, offer the spatial orientation between esophagus and the interested cardiac structure, and thus enable us to foresee whether the desired procedure-specific imaging is feasible or not since each step in our method can be translated back into a patient-specific TEE acquisition protocol. If the procedure-specific imaging is not feasible, the heart-team can promptly use our method to seek surrogate TEE views at other esophageal or transgastric levels. This assists interventional echocardiographers and operators reach to a consensus in the preoperative rehearsal and may even shorten the procedure time and fluoroscopic time.

In this era of echocardiography-fluoroscopic and CT-fluoroscopic fusion imaging, one might underestimate the importance in comprehending the 3D orientation of 2D TEE images. Nevertheless, it is important for transcatheter interventionists to extend their proprioception all the way to the catheter tip and nearby structures. Proprioception needs visual assistance to become precise perception; however, unlike the electroanatomical mapping systems of the ablation catheters, current devices for structural heart diseases are still unable to work on precise targeting or tracking in the 3D space but only projecting to the fluoroscopy where essential spatial resolution is lacking. In fact, simply putting all modalities onto a cine fluoroscopy produces nothing more than confusions because there are either just static segmentations or mere artificial intelligence based modeling rather than imaging. Although fusion imaging with high-resolution TEE images is feasible nowadays, anatomical details are still discernible only when the image intensifier is at the correct projection angles to spread the $2 \mathrm{D}$ images. Our method allows patient-specific fluoroscopic viewing angles to be generated simultaneously during the MPR manipulation. In combination with the concept of the optimal projection curve and the projection-slice relationship, ${ }^{9,10} \mathrm{CT}$ MPR can enhance a better understanding of the spatial orientation of echocardiography-fluoroscopic fusion imaging.

\section{5 - CONCLUSION}

Stepwise cardiac computed tomography manipulation to mimic trans-esophageal echocardiography is the last piece of the puzzle to achieve mental co-registration of the three important imaging modalities. With a 
high quality electrocardiogram gated cardiac CT, we are enabled to foresee the fluoroscopy projections and TEE images in a preoperative rehearsal to decide the interventional fluoroscopy projection angles according to the patient-specific conditions, and to assist the interventional echocardiographer to plan the imaging.

\section{HIGHLIGHTS}

MPR manipulation of the cardiac CT can integrate different imaging modalities in evaluating structural heart diseases and set up the procedural imaging plan in the preoperative rehearsal.

The steps of MPR manipulation of the cardiac CT can be translated back into TEE probe manipulation protocols and thus shorten the learning curve for novices.

Adjusting images of different modalities to similar reference planes and configurations may reduce the discrepancies which appear during device sizing.

\section{CONFLICTS OF INTEREST}

The authors have no conflicts of interest to disclose.

\section{REFERENCES}

1. Doherty JU, Kort S, Mehran et al: ACC/AATS/AHA/ASE/ASNC/HRS/SCAI/SCCT/SCMR/STS 2017 Appropriate Use Criteria for Multimodality Imaging in Valvular Heart Disease. J Am Coll Cardiol. 2017:70:1647-72.

2. Wunderlich NC, Beigel R, Ho SY, et al: Imaging for Mitral Interventions. JACC Cardiovasc Imaging. 2018;11:872-901.

3. Doherty JU, Kort S, Mehran R, et al: ACC/AATS/AHA/ASE/ASNC/HRS/SCAI/SCCT/SCMR/STS 2019 Appropriate Use Criteria for Multimodality Imaging in the Assessment of Cardiac Structure and Function in Nonvalvular Heart Disease. J Am Coll Cardiol. 2019:73:488-516.

4. Jone P-N, Haak A, Ross M, et al: Congenital and Structural Heart Disease Interventions Using EchocardiographyFluoroscopy Fusion Imaging. J Am Soc Echocardiogr [Internet]. 2019 [cited 2019 Dec 3]. Available from: https://linkinghub.elsevier.com/retrieve/pii/S0894731719309101

5. Jone P-N, Haak A, Petri N, et al: Echocardiography-Fluoroscopy Fusion Imaging for Guidance of Congenital and Structural Heart Disease Interventions. JACC Cardiovasc Imaging. 2019:12:1279-82.

6. Gao G, Penney G, Ma Y, et al: Registration of 3D trans-esophageal echocardiography to X-ray fluoroscopy using image-based probe tracking. Med Image Anal. 2012:16:38-49.

7. Arujuna AV, Housden RJ, Ma Y, et al: Novel System for Real-Time Integration of 3-D Echocardiography and Fluoroscopy for Image-Guided Cardiac Interventions: Preclinical Validation and Clinical Feasibility Evaluation. IEEE J Transl Eng Health Med. 2014:2:1-10.

8. Thaden JJ, Sanon S, Geske JB, et al: Echocardiographic and Fluoroscopic Fusion Imaging for Procedural Guidance: An Overview and Early Clinical Experience. J Am Soc Echocardiogr. 2016:29:503-12.

9. Thériault-Lauzier P, Andalib A, Martucci G, et al: Fluoroscopic Anatomy of Left-Sided Heart Structures for Transcatheter Interventions. JACC Cardiovasc Interv. 2014:7:947-57.

10. Pighi M, Thériault-Lauzier P, Alosaimi H, et al: Fluoroscopic Anatomy of Right-Sided Heart Structures for Transcatheter Interventions. JACC Cardiovasc Interv. 2018:11:1614-25.

11. Lu MT, Ersoy H, Whitmore AG, et al: Reformatted Four-Chamber and Short-Axis Views of the Heart Using Thin Section ([?]2 mm) MDCT Images. Acad Radiol. 2007:14:1108-12.

12. Piorkowski A, Kempny A: The Transesophageal Echocardiography Simulator Based on Computed Tomography Images. IEEE Trans Biomed Eng. 2013:60:292-9. 
13. Bax JJ, Debonnaire P, Lancellotti P, et al: Transcatheter Interventions for Mitral Regurgitation. JACC Cardiovasc Imaging. 2019:12:2029-48.

14. Hahn RT, Abraham T, Adams MS, et al: Guidelines for Performing a Comprehensive Transesophageal Echocardiographic Examination: Recommendations from the American Society of Echocardiography and the Society of Cardiovascular Anesthesiologists. J Am Soc Echocardiogr. 2013:26:921-64.

15. Hahn RT, Nicoara A, Kapadia S, et al: Echocardiographic Imaging for Transcatheter Aortic Valve Replacement. J Am Soc Echocardiogr Off Publ Am Soc Echocardiogr. 2018:31:405-33.

16. Prihadi EA, Delgado V, Hahn RT, et al: Imaging Needs in Novel Transcatheter Tricuspid Valve Interventions. JACC Cardiovasc Imaging. 2018:11:736-54.

\section{FIGURE LEGEND}

FIGURE 1 Mathematical coordinate system for different imaging modalities

(A) Computed tomography multi-planar reconstruction is based on Cartesian coordinate system. (B) Fluoroscopy is the projection in the direction of image intensifier, which is of spherical coordinate system. Spider view of left coronary artery is yielded from the left anterior oblique (LAO)/ caudal (CAU) projection. (C)Trans-esophageal echocardiography imaging reference plane is similar to the polar coordinate plane in the cylindrical coordinate system. 1-3= spatial orientation of different TEE sectors $(1=$ Transgastric left ventricular short axis view; $2 \& 3=$ Two orthogonal mid-esophageal views); Blue circle $=$ mid-esophageal level; Orange circle $=$ transgastric level; AsAo = ascending aorta; DsAo $=$ descending aorta; LA = left atrium; $\mathrm{LAD}=$ left anterior descending artery; $\mathrm{LCX}=$ left circumflex artery; $\mathrm{LM}=$ left main trunk; $\mathrm{LV}=$ left ventricle; $\mathrm{LV}=$ left ventricular outflow tract; $\mathrm{RV}=$ right ventricle.

FIGURE 2 Methods of stepwise multi-planar reconstruction (MPR) to simulate trans-esophageal echocardiography (TEE) from cardiac computed tomography (CT)

LEFT: Step 1: Invert the z-axis. Eye symbols and colored human models describe the visual perspectives of the CT slices. After obtaining top visual perspective, crosshairs are moved into the esophageal lumen (shorter yellow arrow). Step 2: Turn to the left. On the axial MPR plane (red box), sagittal MPR line is rotated to cross the center of mitral valve. Slight retroflex is added to avoid foreshortening on 4-chamber view (blue box). Steps 3: Rotate. On coronal MPR plane (green box), axial MPR line is rotated to obtain 2-chamber and 3-chamber (long-axis) view. RIGHT: Similarity in configuration is examined by overlaying the yielded $\mathrm{CT}$ slices with the corresponded TEE views. ALPM = anterolateral papillary muscle; AsAo = ascending aorta; $\mathrm{AV}=$ aortic valve; DsAo = descending aorta; $\mathrm{LA}=$ left atrium; $\mathrm{LV}=$ left ventricle; $\mathrm{MV}=$ mitral valve $\mathrm{PMPM}=$ posteromedial papillary muscle $\mathrm{RA}=$ right atrium; $\mathrm{RSPV}=$ right superior pulmonary vein; RV = right ventricle; RVOT $=$ right ventricular outflow tract.

FIGURE 3 Flex to the right

(A) Orange box: Decomposition of trans-esophageal echocardiography (TEE) probe "flex to the right" movement into displacement and negative rotation of the Cartesian coordinate system.(B) Effect of "flex to the right" on mid-esophageal left ventricle 4-chamber view: the left ventricular outflow tract (LVOT) is revealed due to the "negative rotation" component, and is offset with a compensatory positive rotation. (C) Cardiac tomography multi-planar reconstruction simulation. Without "flex to the right", the line of symmetry of the TEE scanning sector is close to the lateral mitral annulus. As a result, the rotation-yielded bi-commissural view is compromised (left lower green box). With "flex to the right", the line of symmetry of the TEE scanning sector is displaced toward the center of mitral valve, and the LVOT is again revealed due to the "negative rotation" component. Compensatory positive rotation and rotated degree for bi-commissural view are illustrated with the right lower green box, and better bi-commissural view is obtained. AsAo = ascending aorta; DsAo = descending aorta; Eso = esophagus; LA =left atrium; $\mathrm{LV}=$ left ventricle; $\mathrm{LV}=$ left ventricle outflow tract; $\mathrm{RA}=$ right atrium; $\mathrm{RV}=$ right ventricle; $\mathrm{RVOT}=$ right ventricular outflow tract; $\mathrm{S}=$ spine. 
FIGURE 4 Flex to the left with anteflexed echo probe

(A) Orange box: Decomposition of trans-esophageal echocardiography (TEE) probe "flex to the left during anteflex" movement into rotation about the esophagogastric junction and positive rotation of the Cartesian coordinate system. The right colored human models explain the visual perspective of coronal multi-planar reconstruction plane (green box). (B) Effect of "flex to the left during anteflex" on transgastric mitral apparatus view: the left ventricular outflow tract (LVOT) is revealed on the right side. The generated view serves as surrogate image to estimate pressure gradient at LVOT when transgastric LVOT views are not feasible. ALPM = anterolateral papillary muscle; AsAo = ascending aorta; AV= aortic valve; EG junction = esophagogastric junction; LA = left atrium; LAA = left atrial appendage; LPV = left pulmonary vein; $\mathrm{LV}=$ left ventricle; LVOT = left ventricular outflow tract; MDCT = multi-detector computed tomography; $\mathrm{MPA}=$ main pulmonary artery $\mathrm{MPR}=$ multi-planar reconstruction; $\mathrm{MV}=$ mitral valve $; \mathrm{PMPM}=$ posteromedial papillary muscle.

FIGURE 5 Mitral apparatus

Blue box: advance and anteflex of the trans-esophageal echocardiography probe to obtain the transgastric left ventricle short-axis view (blue arrow). Green box: Rotation of the multi-planar reconstruction (MPR) line on the coronal MPR plane to obtain the transgastric 2-chamber view for mitral apparatus (green arrow). $\mathrm{ALPM}=$ anterolateral papillary muscle; DsAo = descending aorta; LA = left atrium; LAA = left atrial appendage; LCX = left circumflex artery; LPV = left pulmonary vein; $L V=$ left ventricle; $M V=$ mitral valve; $\mathrm{PMPM}=$ posteromedial papillary muscle; $\mathrm{RA}=$ right atrium; $\mathrm{RV}=$ right ventricle.

FIGURE 6 Left ventricular outflow tract

UPPER: Standard deep transgastric 5-chamber view without rotation (0 degree). LOWER: Rotation at 135 degree: from computed tomography orientation cube, we can easily understand the orientation is almost to be that from anterior perspective. $\mathrm{AML}=$ anterior mitral leaflet; AsAo= ascending aorta; $\mathrm{AV}=$ aortic valve; $\mathrm{LA}=$ left atrium; $\mathrm{LAA}=$ left atrial appendage; $\mathrm{LV}=$ left ventricle; $\mathrm{LVOT}=$ left ventricular outflow tract; $\mathrm{PA}=$ pulmonary artery; $\mathrm{RA}=$ right atrium; $\mathrm{RAA}=$ right atrial appendage; $\mathrm{RV}=$ right ventricle.

FIGURE 7 Tricuspid leaflets

Interatrial septum avoided at deep esophageal level (yellow box), and tricuspid leaflets investigated without acoustic shadowing. Tricuspid valve and its nearby orientations of nearby cardiac structures are highly variable. As a result, preview with computed tomography multi-planar reconstruction slices can help interventional sonographer to understand better the spatial orientation during procedural trans-esophageal echocardiography imaging. AsAo = ascending aorta; CS = coronary sinus; DsAo = descending aorta; Eso = esophagus; $\mathrm{EV}=$ Eustachian valve; $\mathrm{IVC}=$ inferior vena cava; $\mathrm{LA}=$ left atrium; $\mathrm{LV}=$ left ventricle; $\mathrm{MB}$ $=$ moderator band $\mathrm{PB}=$ parietal band $\mathrm{RA}=$ right atrium; $\mathrm{RCA}=$ right coronary artery; $\mathrm{RPV}=$ right pulmonary vein; $\mathrm{RV}=$ right ventricle.

FIGURE 8 Tricuspid apparatus

Papillary muscle of anterior tricuspid leaflet is continuous with MB. Several small papillary muscles arising from the diaphragmatic right ventricle wall support the posterior tricuspid leaflet. Ant $=$ anterior tricuspid leaflet; $\mathrm{CT}=$ chordae tendineae; $\mathrm{D}=$ diaphragmatic right ventricle wall; $\mathrm{MB}=$ moderator band; $\mathrm{PM}=$ papillary muscle; Post $=$ posterior tricuspid leaflet; $\mathrm{RA}=$ right atrium; $\mathrm{RV}=$ right ventricle; $\mathrm{RVOT}=$ right ventricular outflow tract.

FIGURE 9 Left atrial appendage, aortic valve and interatrial septum

AsAo = ascending aorta; BAV = bicuspid aortic valve; $\mathrm{CS}=$ coronary sinus; DsAo = descending aorta; FO = foramen ovale; IAS = interatrial septum; IVC = inferior vena cava; LA = left atrium; LAA = left atrial appendage; $\mathrm{PA}=$ pulmonary artery; Pec. $\mathrm{m} .=$ pectinate muscles; $\mathrm{RA}=$ right atrium; $\mathrm{RV}=$ right ventricle; $\mathrm{RVOT}=$ right ventricular outflow tract; $\mathrm{SVC}=$ superior vena cava; $\mathrm{TV}=$ tricuspid valve. 


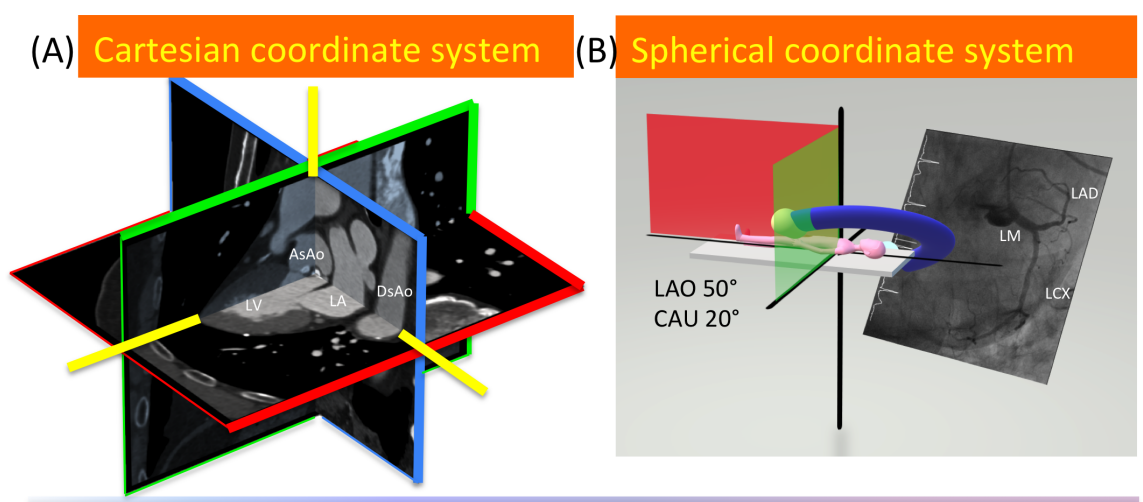

(C) Cylindrical coordinate system
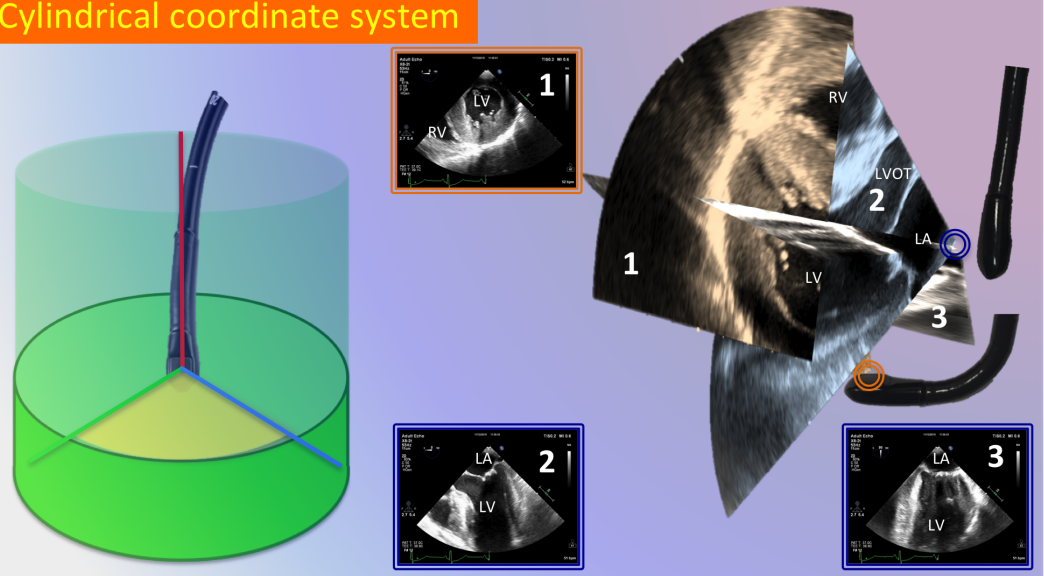


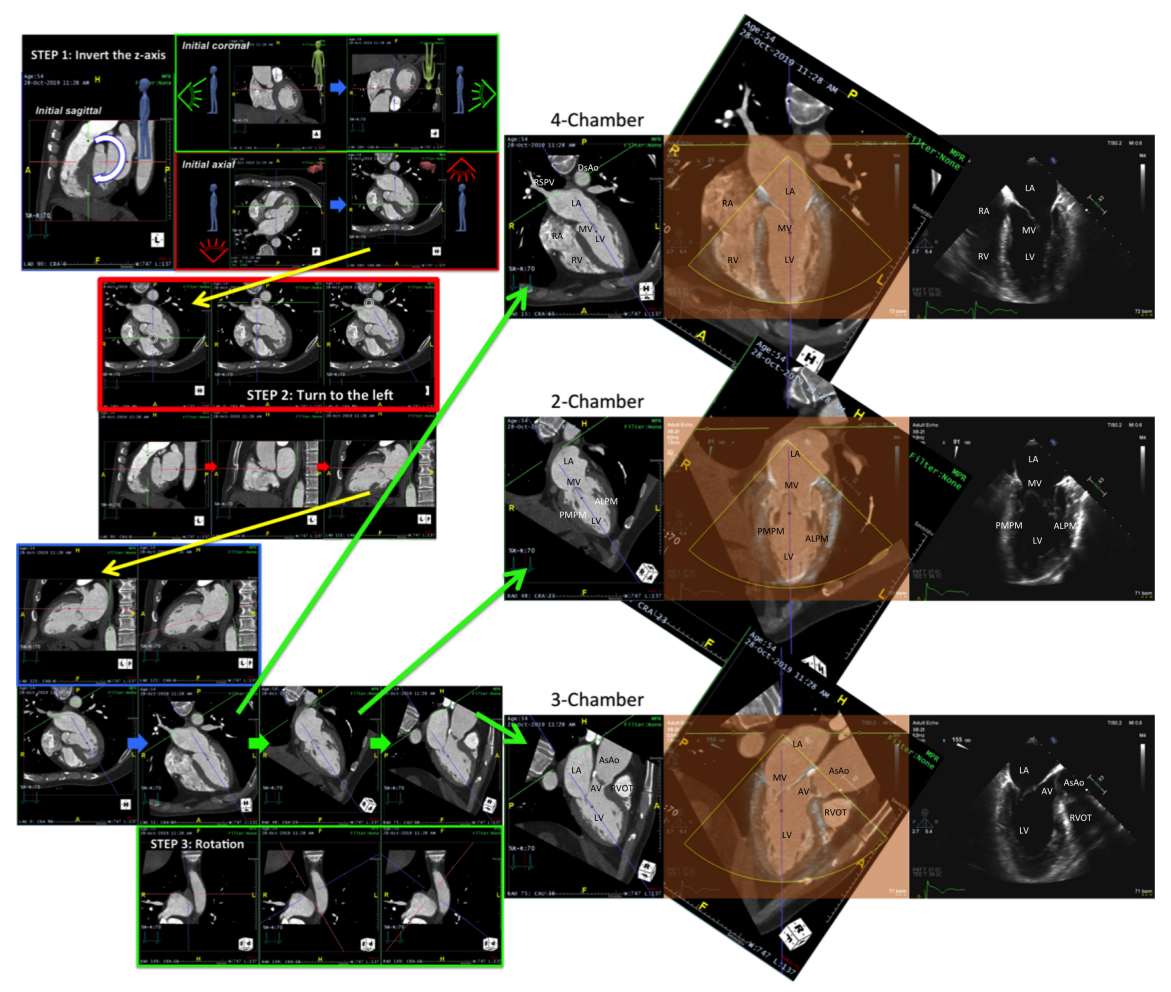



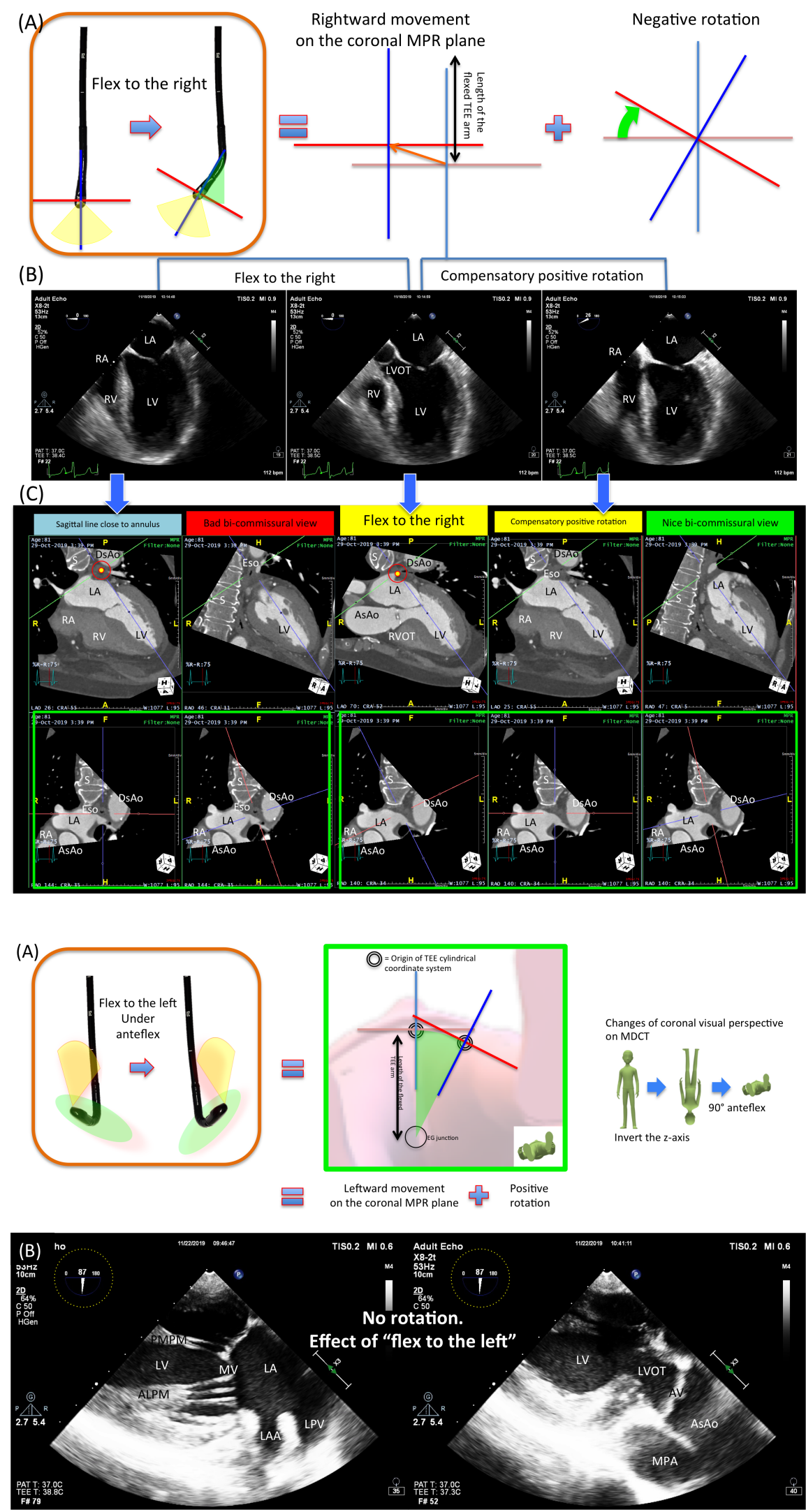

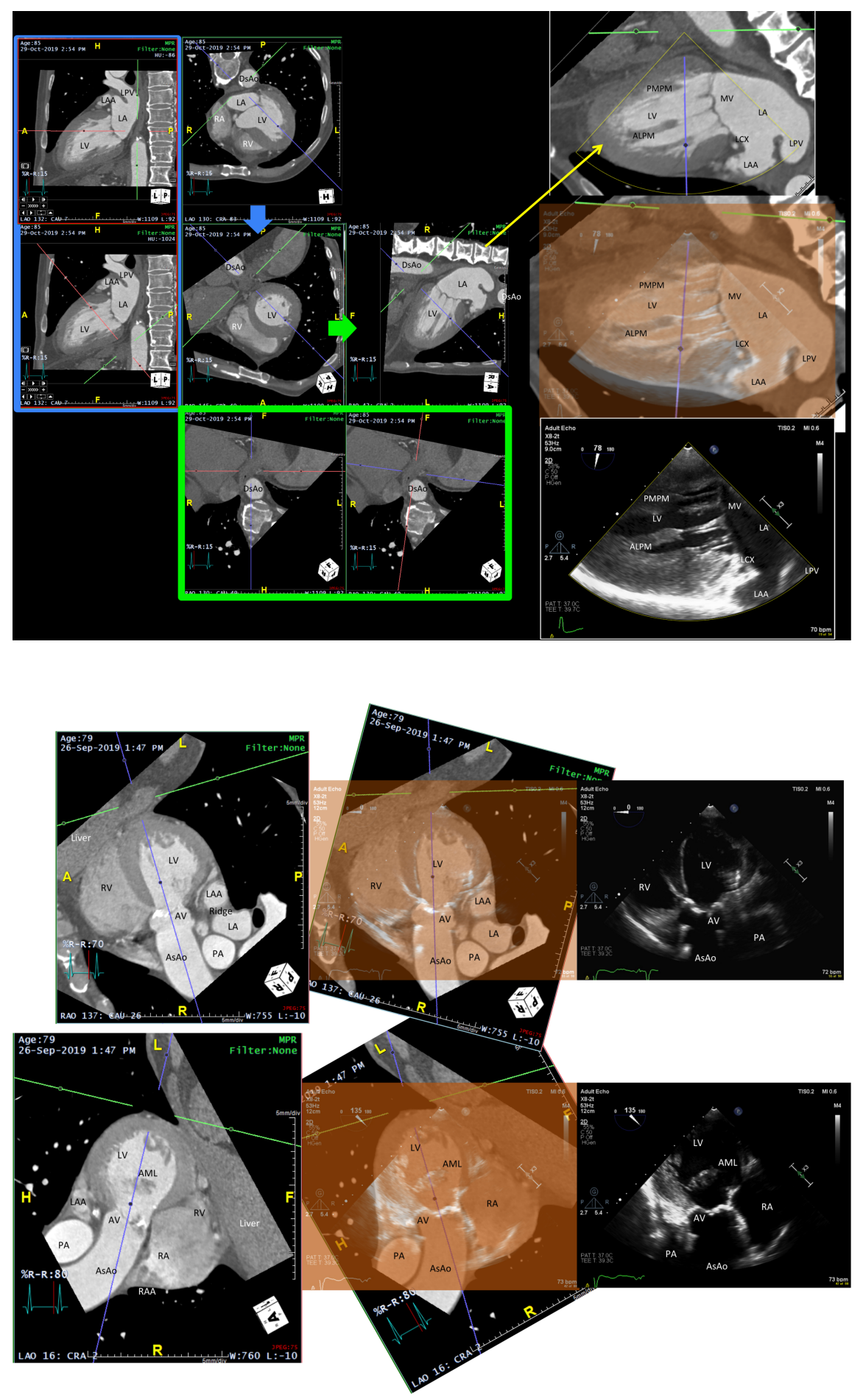

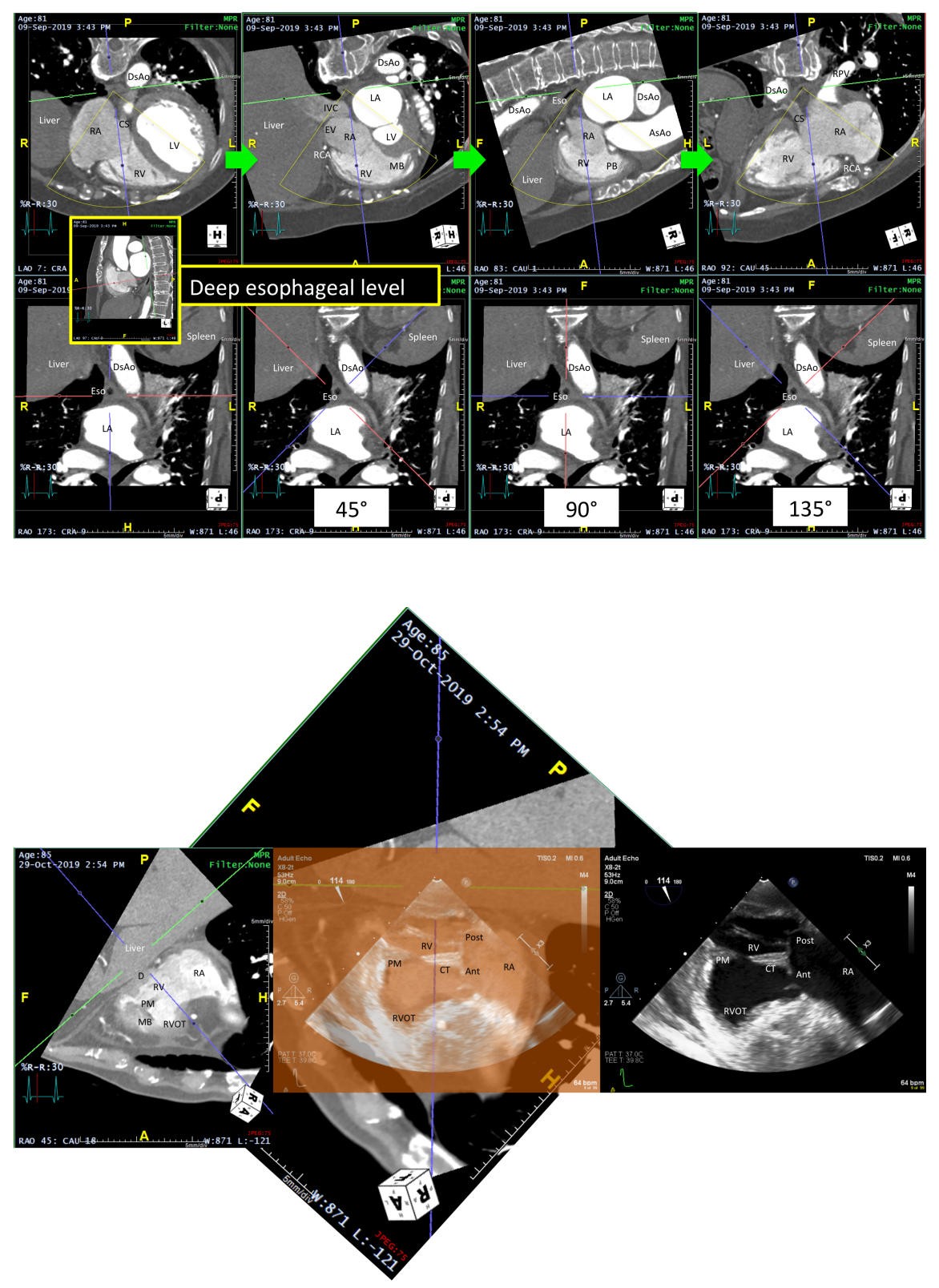


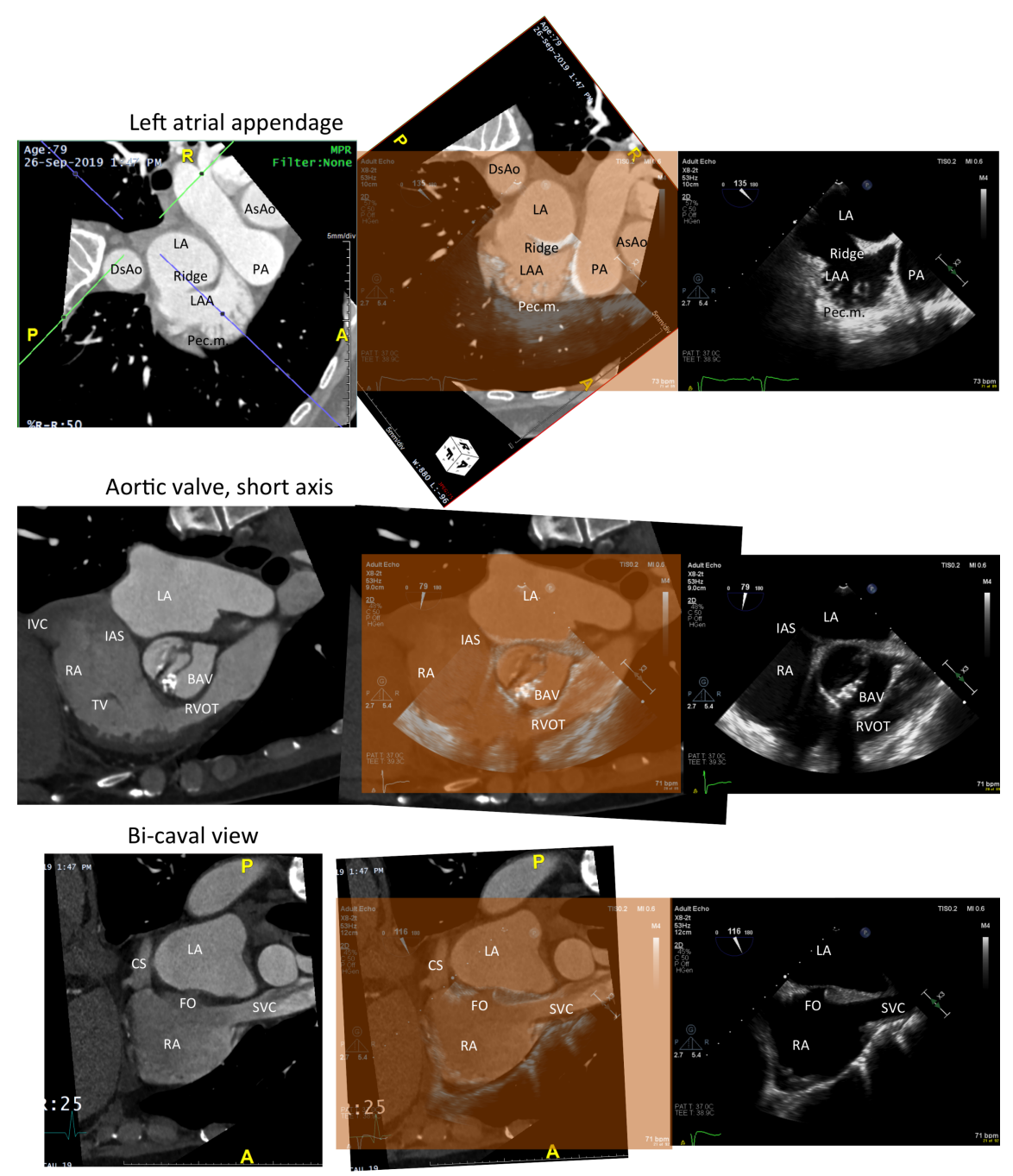

